\title{
Criminologie
}

\section{Le cycle de la violence physique : trajectoire sociale et cheminement personnel de la violence individuelle et de groupe}

\section{Marc LeBlanc}

Volume 23, numéro 1, 1990

Nouvelles violences à l’adolescence

URI : https://id.erudit.org/iderudit/017287ar

DOI : https://doi.org/10.7202/017287ar

Aller au sommaire du numéro

\section{Éditeur(s)}

Les Presses de l'Université de Montréal

ISSN

0316-0041 (imprimé)

1492-1367 (numérique)

Découvrir la revue

Citer cet article

LeBlanc, M. (1990). Le cycle de la violence physique : trajectoire sociale et cheminement personnel de la violence individuelle et de groupe. Criminologie, 23(1), 41-74. https://doi.org/10.7202/017287ar
Résumé de l'article

This article is about interpersonal physical violence. The description of the course of violent crimes in Quebec, based on official statistics and surveys, shows an increase in these crimes and in gang fighting, but the level is equivalent to that we had already known. There are a number of factors responsible for this situation - recent immigration, the reappearance of adolescent gangs and certain social changes. An analysis of interpersonal violence by means of a longitudinal study of individuals shows that it is an activity of the youth, not often repeated and of short duration, which is preceded by other criminal activities. It has been established that there is a strong continuity between agressive acts in early childhood, fighting during adolescence and violent crimes during adulthood. It has also been shown that the appearance of this type of conduct can be predicted under certain conditions. The results call for several preventive measures: the integration of young immigrants, the development of mechanisms for adolescent conviviality, support during the transition from school to the workplace and energetic control of adolescent gangs. Concerning action with regard to individuals, we discuss early discovery of persons at risk and specific types of intervention
Ce document est protégé par la loi sur le droit d'auteur. L'utilisation des services d’Érudit (y compris la reproduction) est assujettie à sa politique d'utilisation que vous pouvez consulter en ligne.

https://apropos.erudit.org/fr/usagers/politique-dutilisation/ 
This article is about interpersonal physical violence. The description of the course of violent crimes in Quebec, based on official statistics and surveys, shows an increase in these crimes and in gang fighting, but the level is equivalent to that we had already known. There are a number of factors responsible for this situation - recent immigration, the reappearance of adolescent gangs and certain social changes. An analysis of interpersonal violence by means of a longitudinal study of individuals shows that it is an activity of the youth, not often repeated and of short duration, which is preceded by other criminal activities. It has been established that there is a strong continuity between agressive acts in early childhood, fighting during adolescence and violent crimes during adulthood. It has also been shown that the appearance of this type of conduct can be predicted under certain conditions. The results call for several preventive measures: the integration of young immigrants, the development of mechanisms for adolescent conviviality, support during the transition from school to the workplace and energetic control of adolescent gangs. Concerning action with regard to individuals, we discuss early discovery of persons at risk and specific types of intervention.

\section{INTRODUCTION : CLARIFICATION DE LA NOTION DE VIOLENCE}

Le terme de violence réfère, si l'on consulte le Robert, à l'utilisation d'une force brutale contre la volonté d'une personne; cette définition est acceptée par les philosophes et les spécialistes des sciences humaines (voir Roy, 1989, et Hébert, 1989). Nous nommerons ce type d'interaction entre un agresseur et sa victime, la violence interpersonnelle. Actuellement, il est à la mode, dans les milieux de l'éducation et des affaires sociales, de même que dans les médias, d'employer le terme de violence pour désigner une réalité beaucoup plus vaste et dont les contours sont plus difficilement délimitables. Un terme d'utilisation aussi générique exige donc quelques clarifications.

* Professeur titulaire, École de psycho-éducation, Chercheur titulaire, Groupe de recherche sur l'inadaptation psychosociale à l'enfance, Université de Montréal. 
D'abord, il faut distinguer entre, d'une part, les attitudes, les valeurs et les mentalités violentes et, d'autre part, les comportements violents. L'énumération des premières pourrait être fort longue: racisme, sexisme, marginalisation des déviants, décalage économique, guerre, violence à la télévision, etc; il s'agit d'autant d'attitudes, de situations et de contextes sociaux qui constituent ou favorisent l'émergence des conduites violentes dans les sociétés contemporaines. Dans cet article, nous nous limiterons aux gestes violents des individus, aux comportements qui impliquent une interaction entre des personnes sur un fond de coercition. Même en se limitant à la violence interpersonnelle, il s'agit d'une réalité fort complexe des points de vue scientifique et social. D'un point de vue scientifique ces conduites ont l'avantage d'être facilement mesurables parce que spécifiques et, d'un point de vue social, elles constituent un phénomène visible, circonscrit et inquiétant et elles exigent une action énergique de protection de la société et d'aide aux auteurs et aux victimes.

La définition des comportements violents ne saurait être complète sans tenir compte de la nature des gestes de violence, aussi convient-il de distinguer au moins cinq sortes d'utilisations de la force dans le cadre des relations interpersonnelles: les agressions symboliques (faire des mimiques, claquer une porte), les agressions verbales (crier, répliquer), les agressions psychologiques (menacer, intimider), les agressions physiques (attaquer, se bagarrer, faire des attouchements sexueis) et les agressions matérielles, la destruction des biens d'autrui (vandaliser la propriété privée ou publique). C'est principalement à la violence interpersonnelle de nature physique que nous nous arrêterons dans ce texte parce que la considération de ces cinq modes d'expression de la violence interpersonnelle nous entraînerait dans des domaines de la connaissance trop diversifiés et d'inégales valeurs.

Par ailleurs, il n'est pas rare que l'on utilise les termes violence et délinquance comme synonymes. Alors, la délinquance acquisitive, les vols de toutes sortes et les commerces illicites (trafic de drogues, prostitution), n'impliquant pas directement l'utilisation d'une menace ou d'une force brutale à l'égard d'une personne, sont rendus équivalents à toutes les formes de violence interpersonnelle que nous venons d'énumérer. Les modes de passage à l'acte étant fort différents entre les délinquances acquisitives et interpersonnelles, et ce au cours de l'adolescence et de la jeunesse (voir Fréchette et Le Blanc, 1987, et Le Blanc et Fréchette, 1989), il y a donc lieu de les traiter séparément. Les différences résident principalement dans l'impulsivité du geste, l'emploi d'instruments et le caractère plus restreint du nombre de complices lorsqu'il s'agit de violence interpersonnelle. 
En nous limitant à la violence interpersonnelle de nature physique, nous diminuons la confusion qu'entretient l'utilisation du terme générique de violence et nous nous adressons à un phénomène social qui accapare, avec raison, l'attention des citoyens et des professionnels. Plus particulièrement, nous nous concentrerons sur deux questions fondamentales mais disparates à première vue: Quelle est la trajectoire sociale de la violence physique au cours des dernières décennies au Québec: augmente-t-elle et/ou change-t-elle de forme? Quel est le cheminement de l'individu qui utilise la violence physique: comment arrive-t-il à ce type de conduite? Quels sont les indices qui permettent de prédire le comportement violent au cours de l'âge adulte?

\section{LA TRAJECTOIRE SOCIALE DE LA VIOLENCE PHYSIQUE}

Si l'on en croit les informations transmises par les journaux et les autres médias au cours de l'hiver 1989, la violence augmente sensiblement au Québec et, plus particulièrement, c'est la violence physique qui exploserait. Durant cette période, il n'y a presque pas eu de semaines où au moins un incident impliquant de la violence physique entre jeunes n'était pas rapporté par les médias. Et ce ne serait pas un phénomène local, il s'affirmerait de la même manière dans les grandes villes canadiennes (Toronto, Vancouver) et américaines. Que nous indiquent les statistiques officielles et les enquêtes sociologiques à ce sujet? Confirment-elles les informations journalistiques?

\subsection{LA VIOLENCE PHYSIQUE EST CYCLIQUE ET ELLE PROGRESSE À COURT TERME.}

Il ne fait aucun doute que la quasi-totalité des sociétés occidentales ont connu une augmentation continue et substantielle de la délinquance des adolescents entre le début des années 1960 et les années 1980, mais l'ampleur de la délinquance des mineurs s'est stabilisée au cours de l'actuelle décennie (Cusson, 1990, décrit bien ce phénomène dans plusieurs sociétés occidentales; voir aussi Fréchette et Le Blanc, 1987, pour des données concernant le Québec). On devrait normalement observer la même trajectoire en ce qui concerne la délinquance avec violence au Québec. Qu'en est-il réellement?

La consultation des données rapportées par Le Blanc (1977) concernant la période entre 1962 et 1974 permet de conclure à une augmentation continue des délits suivants: homicides et tentatives, voies de fait et délits sexuels. Rappelons toutefois que durant cette période la population des jeunes a augmenté substantiellement, c'était l'époque đu «baby boom»; par contre, la proportion que représentent ces délits sur l'ensemble des activités délinquantes des adolescents demeure relativement stable, elle varie annuellement entre $1,27 \%$ et $2,14 \%$ des infractions de toute nature que commettent les 
adolescents. À partir de 1974, Fréchette et Le Blanc (1987) rapportent une augmentation continue mais moins importante des infractions suivantes : homicides et tentatives, voies de fait, délits sexuels et vols qualifiés. Les données rapportées par ces deux études peuvent porter à confusion parce que chacune d'elles délimite différemment la violence physique et qu'elles n'incluent pas un phénomène qui inquiète la population à l'heure actuelle, le port d'une arme offensive.

Figure 1: Pourcentage des infractions avec violence sur les Infractions au Code criminel, Québec: 1962-1988

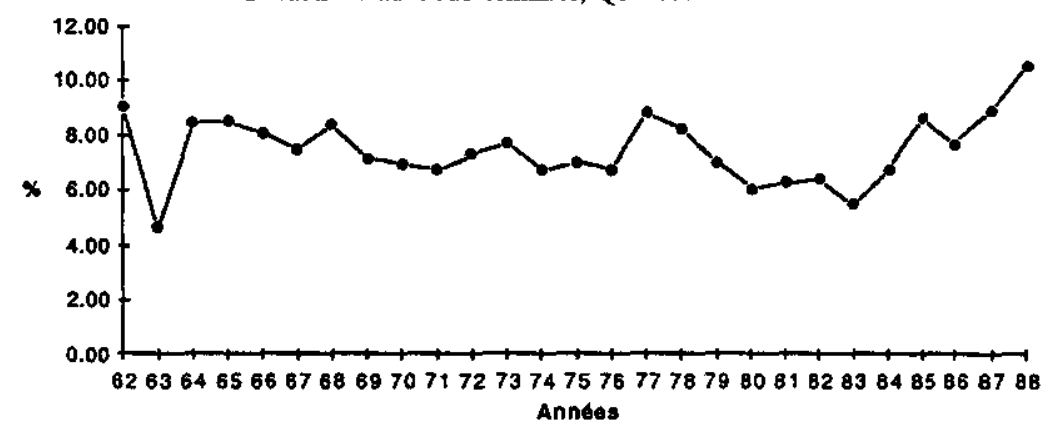

Sources: Statistique Canada (1963-1973), Direction générale de la sécurité publique, Québec (1974-1988).

En conséquence, nous avons cru bon de réanalyser les données sur les infractions avec violence en incluant les homicides et tentatives, les délits sexuels, les vols qualifiés, les voies de fait et le port d'une arme offensive. Analysons l'évolution de cette catégorie d'infractions entre 1962 et 1988, premièrement en termes de proportion par rapport à l'ensemble des infractions au Code criminel pour lesquelles des jeunes du Québec sont inculpés (figure 1) et, deuxièmement, en termes d'importance relative de chacun de ces types à l'intérieur de l'ensemble des infractions avec violence (figure 2).

Au cours des 27 dernières années au Québec, les infractions avec violence représentent en moyenne 7,66 \% de l'ensemble des infractions au Code criminel et l'écart type est de $1,15 \%$, c'est donc dire que la majorité des années affichent une proportion entre $6,5 \%$ et $8,8 \%$. Il convient de noter que cette comparaison implique une source d'erreur minime; en effet, la définition de l'âge de la responsabilité légale a changé au cours de cette période, elle était de 7 ans entre 1962 et 1979 , de 14 ans entre 1979 et 1984 et de 12 ans depuis 1984. Toutefois, ces changements législatifs n'introduisent qu'une distorsion très minime car, comme nous le verrons ultérieurement, il est exceptionnel qu'un jeune de moins de 15 ans soit condamné pour une infraction avec violence. À la lecture de la figure 1 , nous observons donc qu'à la fin des décennies 
1960,1970 et 1980 , un sommet se démarque et son niveau se situe autour de $9 \%$. Une seule année dépasse ce niveau, c'est 1988 avec $10 \%$. En somme, la violence contre les personnes, tout au moins au cours des trois dernières décennies, évolue suivant un cycle marqué par un maximum en fin de décennie. Cette trajectoire est-elle le résultat de la composition variable de la délinquance avec violence?

Figure 2: Proportion que représente chaque type de délits avec violence, Québec, 1962-1988



Sources: Statistique Canada (1962-1973), Direction générale de la sécurité publique, Québec (1974-1988).

Si nous analysons la nature de ces infractions (figure 2), plusieurs observations s'imposent. Une première concerne le port d'une arme offensive; ces délits représentent une proportion minime et relativement stable des délits avec violence au Québec tout au long des 27 dernières années; il est probable qu'ils augmenteront si la surveillance policière s'accroît dans les écoles, les transports en commun et les autres lieux publics ou si une légistation plus restrictive est adoptée à cet égard. Dans cette foulée, une deuxième observation s'impose: la proportion des délits sexuels semble être la résultante d'un changement législatif au début des années 1980 , et cette proportion avait même commencé à diminuer à partir du milieu des années 1970 , vraisemblablement lorsque les avocats de la défense sont devenus de plus en plus nombreux devant les tribunaux pour mineurs. Une quatrième observation a trait au fait que cette évolution des délits sexuels est tout à fait compensée par l'augmentation des voies de fait. Ces dernières progressent proportionnellement à la diminution et à la disparition des délits sexuels; en fait, les accusations pour délits sexuels sont vraisemblablement devenues des voies de fait dans le système de justice des années 1980. Une quatrième et dernière observation concerne les vols qualifiés; ils fluctuent de façon très importante d'une époque à l'autre et en proportion inverse des voies de fait; c'est comme si les contrevenants 
choisissaient l'une ou l'autre modalité d'exprimer de la violence contre les personnes.

En somme, à long terme les infractions avec violence représentent en gros $8 \%$ des délits criminels bon an mal an, tout en oscillant suivant une vague par décennie. Et, selon les périodes, cette masse de délits se répartit alternativement soit sur les vols qualifiés, soit sur les voies de fait; par exemple, la vague des années 1970 coïncidait avec un surcroît de vols qualifiés alors que la vague actuelle correspond à un surplus de voies de fait. Toutefois, à court terme la progression des délits avec violence est surprenante et elle exige une analyse plus serrée.

La figure 3 rapporte le nombre d'inculpations au Québec pour les délits contre la personne, contre les biens, les autres infractions au Code criminel et l'ensemble des infractions criminelles au cours de cette décennie. Ainsi, les délits avec violence sont en progression depuis quatre ans; de 1981 à 1984 ils sont passés de 2095 à 1449 pour remonter progressivement jusqu'à 2185 en 1988, soit 90 de plus qu'en 1981. Comment expliquer ce retour à un niveau antérieur? Plusieurs facteurs peuvent être invoqués: des changements démographiques, législatifs, dans les politiques d'application de la loi, la croissance générale de la délinquance, la prévention.

On ne peut expliquer cette croissance par le facteur démographique puisque la proportion des jeunes qui sont responsables des délits avec violence diminue substantiellement depuis le début de cette décennie. La proportion des jeunes par rapport aux adultes passe de 1 pour 4 en 1981 à 1 pour 9 en 1987 (Langelier-Biron, 1989); la progression de cette diminution reflète probablement le fait que la proportion que représentent les adolescents en regard des adultes diminue comme résultat d'une fécondité plus faible (voir Conseil des affaires sociales, 1989).

Figure 3: Nombre d'infractions au Code criminel au Québec par catégories

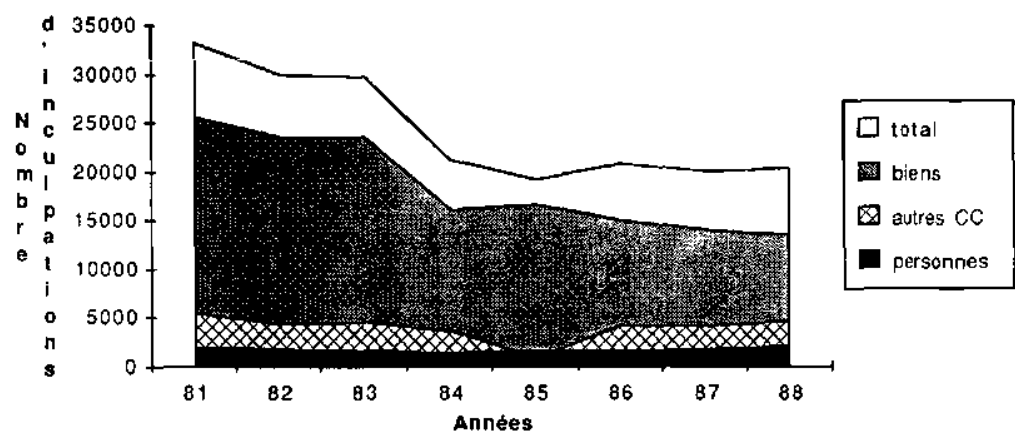

Sources: Direction générale de la sécurité publique, Québec (1981-1988). 
Les changements législatifs ne peuvent être invoqués non plus. Le Code criminel n'a pas été modifié depuis 1984. Au début des années 1980 on avait toutefois changé la définition des délits sexuels ce qui ne peut affecter la progression actuelle, et, depuis 1984, une même loi s'applique aux adolescents, la Loi sur les jeunes contrevenants. Par ailleurs, les politiques d'application de la loi ne sont probablement pas responsables de cette augmentation des délits avec violence. En effet, il n'y a pas eu d'augmentation du personnel policier, les autres infractions au Code criminel demeurent au même niveau, autour de 4000 annuellement, et même l'ensemble de la délinquance demeure stable depuis les trois dernières années, entre 20000 et 21000 inculpations annuellement.

Par contre, ce qui est remarquable, c'est la diminution des délits contre les biens, les vols en particulier. Ils régressent de manière quasi constante entre 1981 et 1988, passant de 25795 à 13688 . Peut-être les programmes des actions préventives, comme Tandem à Montréal, Gros bon sens à Longueuil, etc., ou une situation économique plus favorable sont-ils responsables de la baisse des délits contre les biens? La régression de ce type d'infraction explique sûrement le gain relatif des délits avec violence, mais elle ne peut pas rendre compte du fait que 1988 s'affirme comme l'année qui présente le plus grand nombre de délits avec violence au cours de cette décennie.

En résumé, la proportion que représente la violence physique par rapport à l'ensemble des infractions des adolescents au Code criminel varie cycliquement sur un empan restreint, entre 6,5 et $9 \%$ des infractions criminelles; cette conclusion doit être soulignée malgré une progression à court terme de ce type d'infraction. Cette dernière augmentation, d'une ampleur qui dépasse ce qui a été observé antérieurement, ne semble pas s'expliquer par des facteurs démographiques, économiques, législatifs ou relatifs aux politiques et pratiques d'application de la loi; elle est réelle. Les adolescents d'aujourd'hui adoptent plus fréquemment la violence physique comme modalité d'inadaptation. Cette conclusion est confirmée par nos enquêtes auprès d'échantillons représentatifs d'adolescents en 1974 et en 1985 (Le Blanc et Côté, 1986) et elle s'explique peut-être par la réapparition des bandes d'adolescents qui adoptent un style conflictuel.

\subsection{ELLE PREND PLUS SOUVENT LA FORME DE L'AGRESSION PHYSIQUE EN GROUPE.}

Si nous délaissons les statistiques officielles, qui nous inquiètent et nous rassurent simultanément, pour analyser les résultats de deux enquêtes, distantes de 11 ans, auprès d'échantillons représentatifs d'adolescents montréalais des deux sexes et de 14 et 15 ans (Le Blanc et Côté, 1986), nous notons que, du milieu des années 1970 au milieu des années 1980, la proportion des ado- 
lescents qui participent à des actes d'agression physique demeure stable tandis que cette proportion pour les actes d'agression en groupe augmente (tableau 1).

\section{TABLEAU 1}

Violence physique des adolescents montréalais

telle que rapportée pour la dernière année

1974

1985

violence individuelle

battre quelqu'un qui ne nous a rien fait

menacer

se battre à coups de poing

$7,50 \%$

$7 \%$

$11,50 \%$

$10,80 \%$

$28,40 \% \quad 27,40 \%$

violence de groupe

prendre part à des batailles de groupe faire partie d'un groupe qui fait des coups

$10,30 \%$

$7,20 \%$

$17,50 \%$

$12 \%$

Armes

porter une arme

$21,10 \%$

$26,60 \%$

utiliser une arme en se battant

$4,70 \%$

$4,10 \%$

Si nous gardons à l'esprit que l'âge des adolescents de ces échantillons est justement le moment où la délinquance des adolescents atteint son sommet, tant en termes de participation que de fréquence (voir Fréchette et Le Blanc, 1987, et Le Blanc et Fréchette, 1989), il convient de noter qu'à chaque époque il y a autant d'adolescents qui se battent avec une autre personne ( $28 \%$ en 1974 et $27 \%$ en 1985) et qui utilisent les menaces ( $11 \%$ à chaque époque). Par ailleurs, si le port d'une arme semble augmenter légèrement ( $21 \%$ en 1974 contre $26 \%$ en 1985), les adolescents ne les utilisent pas plus souvent pour se battre avec une autre personne ( $4 \%$ à chaque décennie). Par contre, même en tenant compte du fait que l'écart pourrait être dû au degré de précision des techniques d'échantillonnage, il y a quand même une certaine augmentation des batailles en groupe, elles touchent $17 \%$ des adolescents d'aujourd'hui contre $10 \%$ des jeunes de la décennie antérieure, et en plus, la participation à des groupes qui sont impliqués dans des activités délictueuses, à des bandes de diverses natures au cours de la dernière année, est aussi en progression (11\% maintenant par rapport à 7,2\% avant).

En somme, la délinquance des adolescents, en cette deuxième moitié des années 1980 , se manifeste un peu plus fréquemment par le véhicule de la violence physique et celle-ci semble se produire plus souvent en groupe. 
Si les batailles entre groupes d'adolescents sont plus fréquentes, cela signifie que les victimes sont plus souvent d'autres adolescents et il est alors normal que ces événements ne soient pas toujours rapportés à la police, tout au moins lorsqu'il n'y a pas de blessures. S'il y a plus de conflits entre bandes d'adolescents, cela explique, en conséquence, l'augmentation à court terme des statistiques officielles sur la délinquance avec violence et l'équivalence de la situation actuelle avec le niveau de la violence physique de la fin des années 1970. Mais, même s'il y a plus de conflits entre des groupes d'adolescents, cela ne signifie pas pour autant qu'il y a une augmentation du nombre de bandes à Montréal. Peut-être n'assistons-nous qu'à un changement dans la forme d'expression de la délinquance?

\subsection{LES BANDES CONFLICTUELLES.}

La bande, qu'elle ait des activités déviantes ou pas, est un phénomène normal au cours de l'adolescence depuis au moins les années 1920 (voir Robert et Lascoumes, 1974). La délinquance, pour sa part, est reconnue comme une activité de groupe (voir Reiss, 1986, pour une recension des écrits et Fréchette et Le Blanc, 1987, pour des données québécoises). Par contre, ce qui est particulièrement remarquable depuis quelques décennies, c'est la réapparition en cycles des bandes conflictuelles.

\subsubsection{RÉAPPARITION CYCLIQUE}

En criminologie, beaucoup d'écrits empiriques et théoriques ont été consacrés aux bandes et ce, depuis les années 1920. Pensons à Thrasher (1927), White (1943), Cohen (1955), Cloward et Ohlin (1960), Yablonsky (1962) et la liste s'allonge très rapidement par la suite. Trois sortes de bandes sont distinguées par Cloward et Ohlin (1960) et décrites dans de nombreux écrits empiriques: les bandes conflictuelles qui s'expriment principalement par la violence physique en groupe, les bandes criminelles qui se concentrent sur le vol et les commerces illicites et qui sont plus ou moins intégrées au milieu criminel et les bandes retraitistes qui centrent leurs activités autour de drogues, consommation et commerce. Ces trois types de bandes sont décrits dans les écrits criminologiques depuis les années 1950 dans les grandes villes américaines et anglaises et au début des années 1970 au Québec (Le Blanc, 1971; Le Blanc, Legendre et Ménard, 1973). Ce qui est remarquable, par ailleurs, c'est que même si les bandes retraitistes et criminelles attirent peu l'attention des médias, les professionnels des réseaux de la justice et des affaires sociales savent bien qu'elles sont continuellement présentes dans le paysage de la délinquance; les bandes conflictuelles, par contre, atteignent la notoriété dans les médias de façon cyclique, à chaque décennie elles redeviennent d'actualité. 
Les bandes conflictuelles réapparaissent à l'intersection de deux décennies: à la fin des années 1950 et au début des années 1960 ce furent les blousons noirs qui ont été décrits, entre autres, par Yablonsky (1962) et Racine et al. (1966); à la fin des années 1960 et au début des années 1970 on ne parlait que des «rockers» et surtout des motards (voir Miller, 1975, et Cohen, 1973) qui sont encore d'actualité dans notre monde interlope; puis à la fin des années 1970 et au début des années 1980 les «punks», les «skinheads» et les «hooligans» nous sont venus d'Angleterre (Brake, 1985) et des bandes d'adolescentes violentes ont été recensées au États-Unis (Campbell, 1984); actuellement, les médias d'ici et d'ailleurs rapportent la présence de bandes violentes et les premières publications sortent sur les bandes conflictuelles des années 1980 (Klein, Maxson et Gordon, 1984; Vigil, 1988). En somme, à chaque époque, les bandes conflictuelles arrivent à l'avant- scène, c'est un phénomène social cyclique.

Montréal et le Québec ne sont pas exemptés de ces cycles. Les observateurs les plus anciens de la scène délinquante se rappelleront des "zutsuit» de la fin des années 1940, des "vestes de cuir» de la fin des années 1950, des «motards» de la fin des années 1960 et des «skinheads» et "punks» de l'orée de cette décennie. La bande violente et délinquante n'est pas nouvelle à Montréal. Ainsi à partir des dossiers de la Police de Montréal nous en dénombrions 52 composées de 1038 adolescentset/ou jeunes adultes au début des années 1970 (Le Blanc, Legendre et Ménard, 1973). Parmi ces bandes identifiables par un nom, 30 rassemblaient exclusivement des adolescents de moins de 18 ans, une était composée d'enfants de moins de 12 ans et 21 avaient entre 11 et 134 adolescents comme membres connus des policiers. Ces bandes avaient des contacts avec la police principalement pour des délits contre les personnes et des infractions relatives à l'ordre et à la paix publique. Les journaux de février et mars 1989 ont fait état de l'existence de bandes conflictuelles à Montreal (voir entre autres la série d'articles de Labrosse dans le Journal de Montréal) et selon un communiqué du Service de la police de la CUM il y aurait 27 bandes d'adolescents et jeunes adultes actuellement à Montréal; ce nombre est de beaucoup inférieur au nombre de 52 que nous avions recensées il y a plus de quinze ans.

Notons, en plus, qu'au milieu des années 1970, $62 \%$ des délinquants condamnés par le Tribunal de la jeunesse de Montréal disaient faire partie d'une bande qui était impliquée dans des activités délictueuses, ceci depuis plus d'une année pour $60 \%$ d'entre eux; cette bande avait un chef bien identifié dans $56 \%$ des cas et un rituel précis d'initiation dans une proportion de $35 \%$ d'entre elles (Fréchette et Le Blanc, 1987). Ces adolescents étaient responsables de la proportion élevée de délits avec violence commis pendant 
la deuxième moitié des années 1970 , le sommet du cycle précédent de violence déjà identifié à travers les statistiques officielles.

\subsubsection{AVEC LE SUPPORT DE FACTEURS SPÉCIFIQUES}

Le phénomène des bandes conflictuelles semble donc cyclique. Les délits de violence progressent à court terme en cette fin des années 1980 et les adolescents sont plus nombreux à participer à des batailles en groupe parce qu'ils sont plus nombreux à se rassembler en bande. Comment expliquer cette évolution?

Plusieurs explications sont discutées dans les écrits scientifiques. On peut souligner la diffusion culturelle et sociale grâce aux médias (Cohen, 1973); ils n'auraient aucune influence dans l'apparition de ce phénomène mais ils en favoriseraient l'amplification; toutefois, il faut aussi faire appel à des facteurs beaucoup plus fondamentaux pour expliquer l'émergence de ces bandes.

On retrouve ces bandes conflictuelles principalement dans les milieux défavorisés; ainsi, la dernière recension des bandes à travers les États-Unis (Miller, 1975) montre qu'il est extrêmement rare de trouver des bandes, criminelles ou conflictuelles, relativement organisées dans les milieux aisés, et notre inventaire à Montréal (Le Blanc et al., 1973) montrait qu'elles étaient localisées sur le territoire des postes de police qui desservaient les milieux moins favorisés socio-économiquement.

Elles tendent aussi à fleurir au moment d'une vague d'immigration, cornme le souligne Miller (1975). En fait, il est remarquable de constater qu'en criminologie, les œuvres majeures sur les bandes aux États-Unis rapportent une étude les présentant comme un groupe particulier d'immigrants: dans les années 1920, c'étaient celles associées aux groupes d'immigrants de l'Europe centrale de Chicago que décrivait Thrasher (1927); une décennie plus tard, c'étaient les bandes d'Italiens de Boston que présentait White (1943); au cours des années 1950, c'était le déplacement des Noirs américains du sud vers les villes du nord, et l'arrivée des Portoricains et de leurs gangs était décrite à New York par Yablonsky (1962) et à Chicago par Short et Strodtbeck (1965) et Klein (1971); au cours des années 1970, ce furent les bandes d'origine asiatique de Los Angeles et New York qui attirèrent l'attention (Rice, 1977); et, plus récemment, il s'agissait des bandes associées à l'immigration provenant du Mexique et de l'Amérique centrale décrites part Klein et al., (1984) et Vigil (1988). Au Québec, le phénomène actuel des bandes semble aussi associé à l'immigration récente si l'on s'en rapporte aux médias.

En plus des désavantages socio-économiques et de l'immigration, il faut mentionner les changements dans les contextes de socialisation des adoles- 
cents. Les bandes conflictuelles se manifestent dans les milieux où la monoparentalité est élevée, les écoles détériorées et les activités de loisirs quasi inexistantes (Miller, 1975). Il n'est pas surprenant qu'elles attirent notre attention aujourd'hui au Québec alors que le facteur de la monoparentalité est fort lourd. En effet, notre comparaison de la situation familiale des adolescents en 1974 et en 1985 montre que la proportion des familles monoparentales a augmenté considérablement, passant de $18 \%$ à $26 \%$ (Le Blanc et Côté, 1986). De plus, ces auteurs montrent que cette famille, amputée d'un adulte, comptant de moins en moins d'enfants et de fait plus contrôlante, a pour effet de pousser les adolescents vers ses pairs; ainsi le réseau des pairs s'est agrandi : $53 \%$ des adolescents en 1985 contre $37 \%$ des jeunes en 1974 rapportent avoir quatre amis et plus, et la conformité aux pairs s'est renforcée, $39 \%$ des jeunes d'aujourd'hui contre $32 \%$ de ceux d'hier veulent être comme leurs pairs dans la plupart des choses de la vie. Si les pairs prennent de l'importance, l'école en perd; on n'aime pas plus l'école, on ne s'y sent pas mieux, on ne s'y engage pas plus.

Finalement, un dernier bloc de facteurs peut être invoqué pour rendre compte de la nouvelle manière par laquelle s'exprime la délinquance des adolescents: les transformations des valeurs sociales. Il est bien connu des sociologues que chaque génération réagit aux valeurs mises de l'avant par la génération précédente. Les adultes d'aujourd'hui sont issus de la révolution culturelle de la fin des années 1960 . Celle-ci a mis l'accent sur la tolérance des déviants (l'acceptation des marginaux sur les plans sexuel, du mode de vie, etc.), sur la libération de la femme (la défense de ses droits et de son égalité sociale) et sur la promotion de la personne (son bien-être physique, psychologique et social avant tout). Peut-être que la génération actuelle des jeunes réagit à ces valeurs de leurs parents par des attitudes et des comportements opposés. Ils contestent la tolérance en attaquant brutalement les minorités sexuelles et autres; ils réagissent au féminisme par ce que l'on pourrait nommer le "machisme», le culte de la force brutale et de la domination physique; ils mettent en doute la promotion de la personne au détriment de la vie collective en se tournant vers leurs pairs et en se rassemblant en bandes.

\subsection{CONCLUSION}

Quels que soient les facteurs que l'on avance pour expliquer la nouvelle forme de la délinquance des adolescents, il n'en demeure pas moins qu'en cette fin des années 1980, plusieurs conditions sociales apparaissent favorables à la réapparition des bandes violentes. Et, en effet, les adolescents d'aujourd'hui choisissent plus souvent ce véhicule pour exprimer les difficultés d'adaptation que suppose la transition entre l'enfance et l'âge adulte. Mais de là à conclure qu'il s'agit d'une conflagration de la violence et que nos rues sont 
devenues dangereuses, la marche serait trop large. La progression à court terme de la violence physique dont nous avons parlé, est loin de représenter une quantité effarante et elle compte toujours pour environ $10 \%$ des infractions criminelles, alors que la moyenne sur les 27 dernières années est de $8 \%$. Mais il faut surtout se rappeler que cette progression se fait au profit d'autres formes de délinquance: les délits contre les biens accusent une baisse substantielle durant cette période. On assisterait à une sorte de déplacement de l'activité délictueuse, d'une forme axée sur le vol à une forme centrée sur la violence interpersonnelle.

\section{CHEMINEMENT INDIVIDUEL VERS LA VIOLENCE PHYSIQUE}

Si, au Québec, la violence physique se manifeste au cours de cette décennie suivant des rythmes variables, quel est le cheminement des individus qui la pratiquent? Voilà la deuxième question que nous posons. Plus particulièrement, nous décrirons l'émergence et la continuité de la violence physique dans le répertoire des conduites délinquantes des jeunes, et nous analyserons les facteurs qui permettent de prédire sa présence et son amplification. Mais avant d'aborder ces thèmes, nous décrirons l'ampleur et la nature de cette activité.

En fait, nous analyserons la violence physique produite par un échantillon représentatif d'adolescents montréalais ( 1611 ) recrutés dans tous les milieux sociaux alors qu'ils avaient entre 12 et 16 ans, et celle que commet un échantillon de pupilles du tribunal (470) recrutés au Service de probation ou dans les centres d'accueil alors qu'ils avaient 15 ans en moyenne; il s'agit d'échantillons composés uniquement de sujets mâles. En général les activités délictueuses de ces sujets sont décrites par Le Blanc et Fréchette (1989). Puisque ces individus sont nés entre 1958 et 1962, leur adolescence s'est donc déroulée vers le milieu des années 1970 , au cours de la vague de violence qui a connu son apogée en 1977 et que nous avons identifiée précédemment. De plus, la jeunesse des sujets de ces échantillons s'est répartie sur les années 1980, la période où les délits de violence des adultes sont devenus proportionnellement beaucoup plus nombreux que ceux des adolescents, comme l'établit Langelier-Biron (1989).

\subsection{LA NATURE DE L'ACTIVITÉ CRIMINELLE AVEC VIOLENCE}

Analysons l'ampleur et la nature de la violence physique que pratiquent ces individus. Pour ce faire, nous utiliserons le modèle d'analyse de l'activité délictueuse proposé par Le Blanc et Fréchette (1989), nous référerons en particulier aux paramètres de description et de délimitation suivants : participation, fréquence, variété, nature, précocité, durée et âge d'arrêt. Ces données sont 
présentées au tableau 2 à la fois pour la délinquance officielle des échantillons de la population et du tribunal et pour la délinquance rapportée en entrevue par ce dernier groupe de sujets.

\subsubsection{UNE PARTICIPATION LIMITÉE}

Le Blanc et Fréchette (1989) établissent que $14 \%$ des adolescents et $92 \%$ des pupilles du tribunal sont condamnés pour une infraction au Code criminel avant la fin de la vingtaine. Par contre, comme le montre le tableau 2, au cours de la même période, seulement $3 \%$ des premiers contre $52 \%$ des seconds seront condamnés pour une infraction avec violence (homicides et tentatives, délits sexuels, voies de fait, vols à main armée ou avec violence sur la personne). Le niveau de $3 \%$ dans notre échantillon représentatif de la génération de 1960 à Montréal est semblable au $4 \%$ rapporté pour Stockholm par Wikström (1985); cette proportion est supérieure à ce que comptent Hamparian, Shuster, Dinitz et Conrad (1978) à Columbus, Ohio (1,34 \%), mais elle est de beaucoup inférieure à la proportion en milieu ouvrier à Londres, 12 \% selon Farrington (1989a), et pour les résidants de Philadelphie arrêtés pour un délit avec blessures, 8,6 \% que rapportent Wolfgang, Thornberry et Figlio (1987). La violence physique est somme toute une activité peu prévalente dans la population générale mais que pratiquent la majorité des pupilles du tribunal.

Au tableau 2, il est intéressant de noter que la participation à des délits avec violence physique devient de plus en plus fréquente de l'adolescence à la jeunesse; elle passe de $28 \%$ à $40 \%$ chez les pupilles du tribunal et de 0,6 à $2,7 \%$ dans la population. Par ailleurs, l'écart est quand même mince entre la délinquance officielle et la délinquance cachée (surtout que les entrevues concernant la délinquance cachée ont eu lieu en moyenne à 23 ans alors que la délinquance officielle couvre toute la vingtaine). Ce faible écart entre la participation officielle et rapportée $(51,6 \%$ VS $50,6 \%)$ indique que presque tous les individus qui commettront des délits avec violence à partir du milieu de la vingtaine ont déjà été condamnés pour de tels délits. Mais, en plus, nous pouvons affirmer que presque tous leurs délits sont sanctionnés puisque les médianes de fréquence sont à peu près équivalentes, qu'il s'agisse de la délinquance officielle totale $(5,45)$ ou de la délinquance cachée totale $(5,50)$; mais, comme en font foi les moyennes, un nombre restreint d'individus commettent beaucoup plus de délits avec violence que le nombre de délits pour lesquels ils sont condamnés par un tribunal. De telles données militent en faveur de l'incapacitation sélective puisque le premier délit est habituellement connu et qu'il est généralement suivi de quelques autres. 
TABLEAU 2

La violence physique dans un échantillon de la population et chez des pupilles du tribunal

\begin{tabular}{|c|c|c|c|c|c|c|c|c|c|}
\hline & \multicolumn{3}{|c|}{$\begin{array}{c}\text { adolescents } \\
\mathrm{N}=1611 \\
\text { délinquance ofricielle }\end{array}$} & \multicolumn{6}{|c|}{$\begin{array}{l}\text { pupilles du tribunal } \\
N=470\end{array}$} \\
\hline & juvénile* & adulte** & totale*** & juvénile• & aduite & totale & juvénile* & adulte**** & totale $* * * *$ \\
\hline participation & $0,60 \%$ & $2,70 \%$ & $3 \%$ & $28,10 \%$ & $40 \%$ & $51,60 \%$ & $39,90 \%$ & $46,10 \%$ & $50,60 \%$ \\
\hline \multicolumn{10}{|l|}{ fréquence } \\
\hline médiane & 2 & 2 & 2 & 1,5 & 3 & 3 & 3,35 & 3,43 & 5.5 \\
\hline moyenne & 2 & 3,33 & 3,35 & 3,01 & 4,9 & 5,45 & 27,83 & 25,55 & 50,42 \\
\hline \multicolumn{10}{|l|}{ variété } \\
\hline médiane & 1,43 & 1,15 & 1,17 & 1,12 & 1,44 & 1,42 & 1.16 & 1,4 & 1,43 \\
\hline moyenne & 1,22 & 1,3 & 1,33 & 1,25 & 1,55 & 1,68 & 1,3 & 1,64 & 1,65 \\
\hline \multicolumn{10}{|l|}{ précocité } \\
\hline âge médian & 17 & 21,15 & 21 & 15 & 20,17 & 17 & 14,62 & 18,48 & 15.79 \\
\hline moyen & 16,11 & 22,09 & 20,9 & 14,57 & 21,01 & 17,9 & 14,24 & 19.1 & 16,18 \\
\hline \multicolumn{10}{|l|}{ durée } \\
\hline médiane (ans) & 1 & 1 & 1 & 1 & 2 & 2 & 1.22 & 1,15 & 1,5 \\
\hline moyenne & 1 & 1,19 & 1,28 & 1,29 & 1,94 & 2,52 & 1,37 & 1,25 & 1,82 \\
\hline \multicolumn{10}{|l|}{ arrêt } \\
\hline âge médian & 17 & 23,5 & 23 & 17 & 25 & 23.5 & 15,62 & 21,59 & 20,08 \\
\hline moyen & 16,86 & 23,79 & 23,17 & 16,1 & 24,4 & 22.57 & 15,51 & 21,46 & 19,37 \\
\hline
\end{tabular}

* juvénile: période de 7 à 17 ans

** adulte: période de 18 à 30 ans

*** totale: période de 7 à 30 ans

- juvénile: période de 0 à 17 ans

- aduite: période de 18 à 23 ans (incluant les déférés)

cotale: période de 0 à 23 ans 


\subsubsection{UNE ACTIVITÉ SIGNIFICATIVE MAIS DE COURTE DURÉE ET TARDIVE}

Sur le plan épidémiologique, ils ne sont donc que $3 \%$ de la population à être impliqués dans de la violence physique. Ces 48 individus sur 1611 sont responsables de 161 délits avec violence, soit en moyenne 3,3 (médiane 2). Quarante-deux pour cent de ces individus sont des délinquants occasionnels tandis que les autres commettent entre 2 et 25 infractions avec violence. Wikström (1985) rapportait pour Stockholm $58 \%$ de délinquants occasionnels. L'activité criminelle avec violence de l'échantillon représentatif de la population masculine de Montréal est tardive puisqu'elle débute en moyenne à 21 ans (médiane 20,94), elle est peu variée puisque les contrevenants commettent un type de délit en moyenne (médiane 1,43), elle est de courte durée puisqu'elle s'étend en moyenne sur 1,28 année (médiane 1) et elle s'arrête donc autour de 23 ans (médiane 23). Ces données sont tout à fait comparables à celles que rapporte Wikström (1985): un début à 19 ans et une durée d'une année et demie.

Même dans notre échantillon clinique, les pupilles du tribunal, la violence physique se manisfeste sous les mêmes traits mais avec une ampleur quand même accentuée. Cinquante-deux pour cent des pupilles du tribunal ont commis au moins un délit avec violence; ils sont responsables de 1325 infractions, soit en moyenne 5,5 (médiane 3), et seulement $26 \%$ sont des délinquants occasionnels, les autres ont perpétré entre 2 et 49 délits avec violence. À titre de comparaison, Miller, Dinitz et Conrad (1982) rapportent que les 1591 sujets de leur échantillon national de délinquants américains qui commettent des délits avec violence avaient été arrêtés 12527 fois pour de tels délits, soit une moyenne de 8 fois (nous avons une moyenne de 5,5 condamnations), et que $27 \%$ étaient des délinquants occasionnels (nous avons $26 \%$ ). En fait, $20 \%$ des pupilles du tribunal ont commis $65 \%$ des infractions avec violence et ils ont commis en moyenne une dizaine d'autres infractions criminelles selon Le Blanc et Fréchette (1989).

La persistance dans la violence de la part des pupilles du tribunal peut s'évaluer par la fréquence, mais aussi par la durée. En général, selon les données rapportées par Le Blanc et Fréchette (1989), l'écart entre le premier et le dernier délit est en moyenne de 5,23 ans alors que pour les infractions avec violence la durée moyenne est de deux ans et demi (médiane 2) (tableau 2). Ces données ne signifient pas que ces individus sont actifs pendant toute cette période, mais il s'agit plutôt du nombre d'années sur lesquelles s'échelonnent les quelques délits avec violence qu'ils commettent. Pendant cette période active, les pupilles du tribunal produisent une médiane annuelle de 1,29 délits avec violence, mais il y a quand même des écarts importants d'une année à l'autre comme le montre la figure 4. Deux sommets sont observables, 
19 ans avec 2,5 délits avec violence et 26 ans avec 1,94 délits de ce type. En fait, la fréquence progresse jusqu'au premier sommet, elle diminue à un niveau inférieur au début de l'adolescence, puis une seconde vague se produit au milieu de la vingtaine. Il est probable qu'il s'agit des mêmes sujets puisque les récidivistes sont prédominants parmi les pupilles du tribunal.

Figure 4: Fréquence annuelle de délits

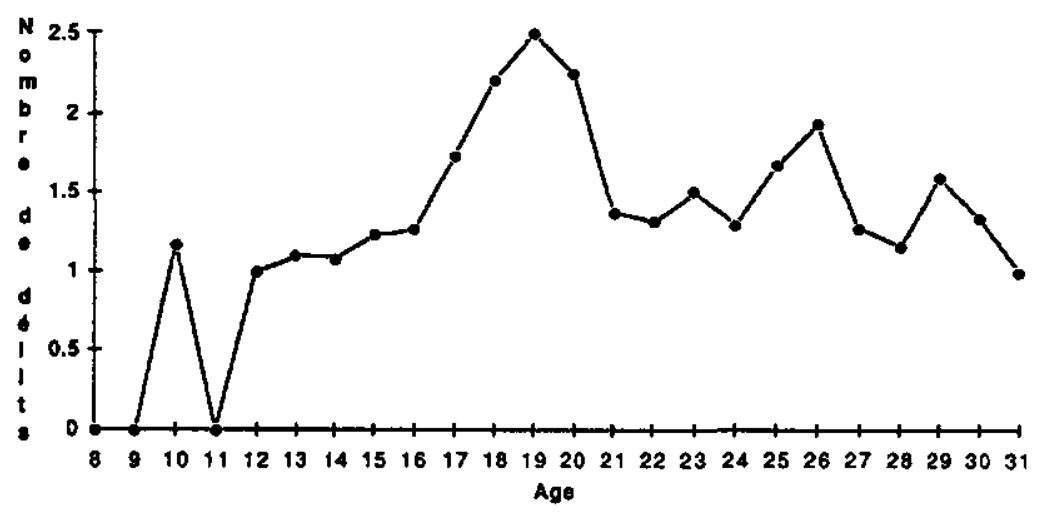

Si les pupilles du tribunal, qui pratiquent les infractions avec violence, tendent à récidiver sur une période de temps relativement courte, il faut signaler que ces activités sont tardives en comparaison de leurs autres activités délictueuses. En effet, la première condamnation pour une infraction au Code criminel a lieu à 14,61 ans; par contre la première condamnation pour un délit avec violence a lieu à 17 ans (médiane 17,89). Notons qu'au tableau 2, l'écart qui s'élargit de l'adolescence à la jeunesse entre le début rapporté de cette activité et le début officiel - au moins une année de différence — reflète le fonctionnement du système judiciaire puisque le calcul s'effectue sur la date de la condamnation et non pas sur la date effective du délit. Signalons aussi que l'âge modal du début est de 15 ans en ce qui concerne les condamnations des pupilles du tribunal alors qu'il est de 16 pour les arrestations à Philadelphie (Wolfgang et al., 1987).

La violence physique est donc un type d'activité délictueuse qui tend à émerger à la toute fin de l'adolescence, et qui par sa durée se situe à cheval sur l'adolescence et la jeunesse. L'âge d'arrêt de ce type d'infraction illustre bien cette observation: il est de 23 ans. C'est aussi une activité récurrente chez les pupilles du tribunal. 


\subsubsection{EN RAPPORT AVEC LE VOL}

Les délits avec violence physique sont relativement nombreux, ils constituent environ le tiers des condamnations; mais, indépendamment du fait qu'il s'agit de délits impliquant l'utilisation de la force, ces activités se présentent comme principalement reliées à l'acquisition de biens et, surtout, d'argent comptant. En effet, les vols qualifiés et avec violence sont les plus répandus, $26 \%$ des pupilles du tribunal les pratiquent; ensuite il s'agit d'assauts, $18 \%$ des sujets de l'échantillon ont été condamnés pour ce type de délit; et finalement, les homicides et les délits sexuels sont rares avec une participation de $4,4 \%$ et de $0,2 \%$ respectivement. Maintenant, si l'on calcule ces proportions seulement sur les sujets qui commettent des délits de violence, elles sont de $51 \%$ pour les vols avec violence, de $35 \%$ pour les assauts, de $9 \%$ pour les homicides et tentatives et de $0,4 \%$ pour les délits sexuels.

Ces délits, par contre, changent de forme entre la première et la seconde moitié de l'adolescence et le début de la jeunesse, comme le montrent Le Blanc et Fréchette (1989). Les vols qualifiés sont plus souvent commis avec un instrument, en employant de la violence physique et de l'intimidation; ils sont mieux planifiés et moins souvent commis sous l'effet de l'alcool ou d'une drogue illicite; en fait, ils sont par ces caractéristiques plus dangeureux. Quant aux voies de fait, elles sont plus impulsives; elles sont plus souvent commises sous l'effet d'une substance intoxicante, avec des complices et en employant une force physique directe parce que les instruments sont moins présents; elles sont souvent plus reliées à un style de vie associé à la fréquentation des débits de boissons alcoolisées.

En résumé, bien que la violence physique se caractérise par de la répétitivité et un accent sur le vol, il n'en demeure pas moins que ce type d'activité délictueuse apparaît tardivement et ne se maintient dans le répertoire comportemental de l'individu que sur une courte période de temps, même si sa dangerosité augmente avec l'âge du délinquant.

\subsection{CONTINUTÉ DE LA VIOLENCE PHYSIQUE}

Après description et délimitation de la violence physique dans la vie des individus, deux questions méritent une attention particulière. Comment se manifeste la continuité de cette violence? Et comment, alors, identifier les étapes comportementales qui conduisent à ce type de conduite?

Depuis la recension d'Olweus (1979), qui analyse 16 études sur les conduites agressives, il est accepté que ce mode de comportement est relativement stable à travers le temps chez les individus. Olweus rapporte une corrélation moyenne de 0,68 entre des mesures des conduites agressives, cofficient qui décroît avec le nombre d'années d'écart entre les mesures. Depuis, d'autres 
travaux sont venus confirmer la stabilité du comportement agressif (voir la recension de Loeber et Southamer-Loeber (1987) et les données récentes de Farrington (1989a, b). Les études abordent toutefois cette question à partir de mesures diverses: évaluation par les pairs, par les parents, auto-évaluation, délinquance officielle. Malgré cette diversité, et surtout malgré un écart temporel quelquefois très grand entre les mesures, les coefficients de stabilité sont impressionnants. Husemann, Eron, Lefkowitz et Walder (1984) rapportent un coefficient de stabilité de 0,46 entre les conduites agressives à 8 ans et à 30 ans chez les mêmes sujets et de 0,58 entre les conduites agressives des parents du sujet à 30 ans et celles du sujet au même âge, et finalement, de 0,65 entre les conduites agressives du sujet à 8 ans et celles de ses enfants au même âge.

S'il y a stabilité des conduites agressives, peut-on parler de continuité parmi les conduites inadaptées? Si la stabilité réfère au fait qu'un comportement d'une mêrne nature, comme les conduites agressives, est répété à différentes phases de la vie, la continuité, pour sa part, se rapporte au fait que deux comportements définis comme inadaptés se suivent dans le temps dans le répertoire comportemental du même individu. La recension de Loeber et Le Blanc (1990) établit que la continuité est courante entre les problèmes du comportement à l'enfance et à l'adolescence et à l'adolescence et à l'âge adulte. Cette continuité s'observerait aussi entre les conduites délinquantes à l'enfance, à l'adolescence et durant la jeunesse (voir aussi Le Blanc et Fréchette, 1989). Regardons quelques données québécoises concernant cette continuité.

Tremblay et Desmarais-Gervais (1986) analysent les rapports entre l'agressivité en maternelle évaluée par le professeur, les pairs et par soi-même et deux critères d'inadaptation: avoir un dossier ultérieur au Tribunal de la jeunesse et la délinquance autorapportée au milieu de l'adolescence. Dans cet échantillon d'enfants provenant d'écoles de milieux ouvriers et défavorisés à la CECM, il est possible de prédire ces deux critères de la mésadaptation avec un degré intéressant de justesse. En ce qui concerne le fait d'avoir ou de ne pas avoir un dossier ouvert devant le Tribunal de la jeunesse, l'échelle d'agressivité à l'évaluation par les pairs permet de prédire de façon concluante la présence d'un dossier de protection pour les filles et la présence d'une condamnation en vertu de la Loi sur les jeunes contrevenants pour les garçons. Ce dernier résultat confirme l'étude de Roff et Wiat (1984). Pour ce qui est de la délinquance autorapportée, les résultats sont très concluants pour les comportements d'agression (se battre, menacer) (significatif à $\mathrm{p}=0,001$ ), concluants pour l'échelle générale de délinquance (significatif à $\mathrm{p}=0,01$ ), mais moins constants pour les formes spécifiques de délinquance (petit vol, vol grave, vandalisme). Ce qui est encore plus remarquable, comme le 
montrent Tremblay, Le Blanc et Schwartzman (1988), c'est que les prédicteurs ne sont pas les mêmes pour les enfants de chaque sexe; pour les garçons, il s'agit de l'agressivité rapportée par les pairs ou le sujet lui-même, tandis que pour les filles l'évaluation par l'enseignante améliore de beaucoup la classification obtenue par le sujet ou les pairs. Farrington (1989a, b) est aussi en mesure de montrer la continuité entre les conduites turbulentes à 8 ans et les bagarres rapportées par le sujet au cours de l'adolescence et de l'âge adulte et entre la turbulence au cours de l'enfance et les condamnations pour une infraction avec violence.

Si nous avons pu documenter une continuité comportementale entre les conduites agressives au début de la scolarisation et au milieu de l'adolescence, des travaux montrent que la prédiction des activités délictueuses à 10 ans peut encore être améliorée si l'on considère la stabilité temporelle des conduites agressives, leur répétition au cours des années du début de l'enfance (Tremblay, Loeber, Gagnon, Charlebois, Larivée et Le Blanc, en préparation) ou la stabilité situationnelle des conduites agressives, le fait qu'elles se reproduisent à l'école, dans la famille et au laboratoire (Charlebois, Le Blanc, Tremblay, Gagnon et Larivée, en préparation). Puisqu'il y a un degré fort important de continuité entre les conduites agressives au cours de l'enfance et les activités délictueuses au cours de la latence et de l'adolescence, attardons-nous à la continuité entre la violence physique à l'adolescence et au cours de la jeunesse telle que recensée par les condamnations par un tribunal.

Rappelons qu'il s'agit d'une conduite qui apparaît relativement tard dans le répertoire des conduites délinquantes des individus et que $48 \%$ des pupilles du tribunal ne sont pas encore impliqués dans des délits avec violence à la fin de la vingtaine. Vingt-quatre pour cent commencent seulement à l'âge adulte tandis que parmi ceux qui ont été condamnés pour un délit avec violence en tant que mineurs $(132 / 470), 60 \%$ récidivent avec un délit de violence après 18 ans $\left(X^{2}=26,87, \mathrm{dl}=1, \mathrm{p}<0,001\right)$. De fait $17 \%$ de l'ensemble des pupilles du tribunal affichent une activité délictueuse avec violence avec continuité de l'adolescence à la jeunesse, et il faut ajouter à cela que la fréquence moyenne de ces délits est de 3 . La considération de la délinquance rapportée par le sujet change peu ces chiffres: $21 \%$ commencent après 18 ans, $62 \%$ de ceux qui avaient commis ce type de délit au cours de l'adolescence le répètent pendant la jeunesse et $25 \%$ des sujets affichent une conduite de violence stable d'une phase de la vie à l'autre. Wolfgang et al. (1987) rapportent aussi une continuité élevée des délits impliquant des blessures, une probabilité de 0,19 ; c'est le niveau le plus élevé parmi les délits criminels («index offenses»). Et Hamparian, Davis, Jacobson et McGraw (1985) rapportent aussi que les 
délits avec violence au cours de l'adolescence prédisent cette conduite au cours de l'âge adulte.

En somme, la commission d'un délit de violence n'est pas un évênement unique, elle est suivie d'au moins quelques autres, et en particulier si le premier a été commis au cours de l'adolescence. La question qui nous intéresse maintenant est la suivante: la violence physique est-elle une étape ou un épisode de la carrière criminelle? C'est un épisode pour le quart des pupilles, comme nous l'avons signalé précédemment en rapportant le nombre de délinquants occasionnels. Par contre, il s'agit d'une marche supplémentaire dans la progression de la carrière criminelle pour les trois quarts des pupilles du tribunal.

Le Blanc et Fréchette (1989) montrent qu'il existe une séquence dans l'apparition des formes spécifiques de la délinquance autorapportée en fonction de l'âge. Cette séquence développementale compte cinq stades dont les deux derniers, la conflagration et le débordement, rassemblent les délits avec violence; les assauts, les délits sexuels et les vols avec violence se situent au stade de la conflagration et les homicides au stade du débordement. Ces auteurs établissent à $55 \%$ la proportion des pupilles du tribunal qui progressent jusqu'au stade de la conflagration, le premier stade incluant les délits avec violence.

Si nous nous limitons maintenant à la délinquance officielle, ces auteurs montrent que ces stades sont réduits à deux (figure 5), les délits contre la propriété qui apparaissent au cours de l'adolescence et les délits contre les personnes qui émergent surtout au tout début de l'âge adulte. Quarante pour cent des pupilles du tribunal passent des délits contre la propriété aux délits avec violence entre l'adolescence et la jeunesse, alors que les autres se limitent à une seule forme de crimes ou combinent les deux au cours de ces deux périodes.

Il est intéressant de noter que seulement $5 \%$ des pupilles du tribunal ( $1 \%$ pendant l'adolescence et $4 \%$ au cours de la jeunesse) se spécialisent dans les infractions avec violence, ils ne sont jamais condamnés pour des infractions contre la propriété. Les autres commettent plus d'un délit avec violence, mais ils sont aussi très productifs. En ce qui concerne les infractions sans violence physique, ils commettent en moyenne 19 délits de ce type au cours de leur carrière (médiane 16). La commission de délits avec violence s'affirme donc comme l'aboutissement d'une carrière criminelle, une étape ultime qui n'est pas inévitable mais qui est relativement courante.

Ces données sur la continuité entre les conduites agressives et les délits avec violence donnent crédit à l'hypothèse de Loeber (1985) à l'effet qu'il existe deux cheminements vers la délinquance chronique: le cheminement 
Figure 5: Délinquance officielle, gradation des types de délits en fonction de l'âge du début et de la durée

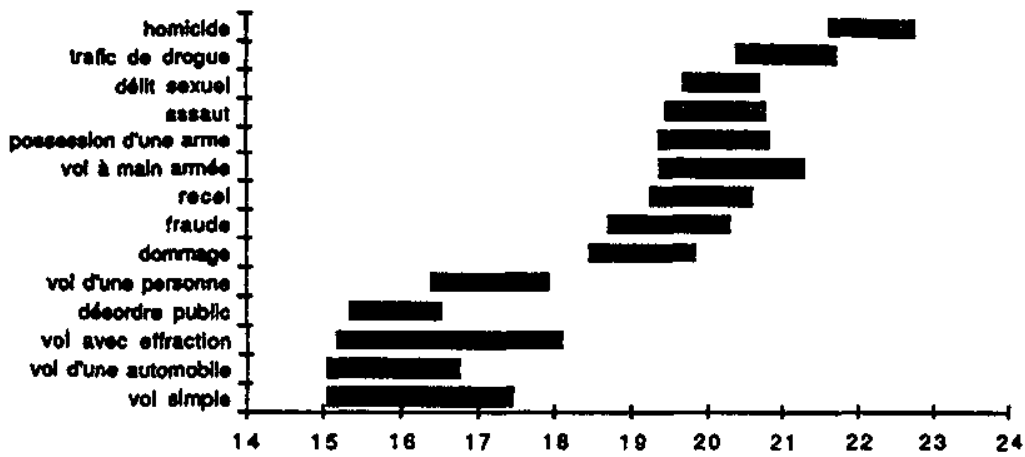

comportant des conduites agressives et celui n'incluant pas de délits avec violence. Chacun de ces cheminements débuterait par des comportements d'opposition au cours de la petite enfance, il comprendrait des conduites agressives au cours de l'enfance et une délinquance diversifiée au cours de l'adolescence; l'aboutissement de ces deux cheminements serait la délinquance chronique; par contre dans le premier cas il y aurait de la délinquance contre les personnes alors que dans le second cas les individus se limiteraient aux infractions contre les biens.

\subsection{UN PRONOSTIC POSSIBLE}

Maintenant que la dynamique comportementale est mieux éclairée, deux voies s'offrent à nous. Premièrement, essayer de répertorier les facteurs, les causes de cette continuité des conduites agressives; une telle démarche nous entraînerait dans une jungle inextricable dont il serait difficile de sortir avec des conclusions claires. Deuxièmement, identifier les marqueurs qui permettraient de reconnaître les individus à risque de présenter une conduite agressive plus fréquente ou de participer à des délits avec violence. Nous optons pour la seconde voie en tentant de répondre à la question suivante: quelles sont les caractéristiques de l'individu et de son milieu, à chaque âge, qui permettent de prédire l'apparition de la violence physique dans son répertoire comportemental?

\subsubsection{DANS CERTAINES CONDITIONS}

La manière de formuler la question limite grandement les études que nous devrons considérer. Ainsi, toutes les études sur la prédiction de la récidive chez les délinquants violents sont d'office éliminées parce qu'elles ne concernent pas l'apparition de novo de la violence physique. Les travaux qui se servent de mesures génériques de l'agressivité seront d'emblée mis de côté 
parce qu'il s'agit d'un trait plutôt que de comportements. Deux types de travaux seront considérés, ceux qui utilisent comme critère soit la délinquance officielle, soit la délinquance racontée; donc, qui emplojent une mesure qui se limite uniquement à des gestes envers d'autres personnes, agressions sexuelles, attaques, bagarres, etc. Qu'il s'agisse de l'une ou l'autre de ces mesures de l'activité délictueuse avec violence, deux observations fondamentales s'imposent.

Premièrement, les travaux qui considèrent plusieurs points de départ à la prédiction, qui utilisent les prédicteurs à un âge antérieur et à plusieurs âges ultérieurs, concluent que les marqueurs les plus efficaces se retrouvent dans la phase de la vie précédant immédiatement celle où apparaît la violence physique. Farrington (1989a) utilise des prédicteurs recueillis à $8,10,14$, 16 et 18 ans et il démontre que les marqueurs les plus puissants se retrouvent toujours dans la phase de la vie qui précède immédiatement celle où le critère est mesuré. Par exemple, Farrington (1989a) montre que les prédicteurs les plus puissants de la violence physique autorapportée (les bagarres) entre 16 et 18 ans sont des variables mesurées au cours de la première partie de l'adolescence, entre 12 et 14 ans. Pour sa part, la violence physique après 18 ans est prédite avec plus d'efficacité grâce aux variables mesurées entre 16 et 18 ans et ainsi de suite pour les autres prédictions qu'il entreprend. Observation que nous avons aussi faite dans nos travaux sur la prédiction de la criminalité adulte en général et la criminalité de violence en particulier, en nous servant des échantillons représentatifs de la population et des pupilles du tribunal que nous avons présentés précédemment (Le Blanc, Hébert et David, 1988); ainsi, la délinquance officielle après 18 ans est prédite le plus efficacement seulement grâce aux variables mesurées au cours de la deuxième moitié de l'adolescence, celles de la première moitié n'améliorant pas la prédiction.

Deuxièmement, une autre observation ressort des études qui tentent de prédire la délinquance en général et la violence physique selon la recension de Loeber et Southamer-Loeber (1987): le comportement inadapté antérieur est le meilleur prédicteur du comportement délinquant ultérieur, il surpasse toujours les variables relatives au milieu social et aux caractéristiques de la personne. C'est aussi ce que nous avons obtenu dans nos travaux sur la prédiction de la criminalité, de la violence et de l'adaptation adulte en général (Le Blanc et al., 1988). Mais, il faut noter, à la lumière des travaux de Farrington (1989a), que le comportement lointain, par exemple au cours de l'enfance, perd de son importance à mesure que des prédicteurs mesurés à un âge ultérieur sont introduits dans l'équation de prédiction, mais, malgré cela, ce prédicteur demeure toujours utile. 
Ces deux observations, considérer avant tout la phase de la vie qui précède immédiatement l'apparition des gestes délictueux avec violence et tenir compte des conduites d'agression et de délinquance, constituent des conditions spécifiques dont on doit tenir compte lorsque l'on recherche les indices pertinents pour le pronostic de la délinquance avec violence. Identifions maintenant les marqueurs connus de ce type de conduite. Pour ce faire, nous traiterons d'abord de l'agression physique rapportée par le sujet lui-même et ensuite des délits avec violence pour lesquels une personne est condamnée.

\subsubsection{LES MARQUEURS}

Farrington (1989a) prédit la violence physique autorapportée, l'implication dans des bagarres, à partir de variables nombreuses qui sont mesurées à partir de 8 ans et ensuite à $10,12,14,16$ et 18 ans. À l'aide de ces données, il identifie les marqueurs des conduites de violence physique entre 12 et 14 ans, entre 16 et 18 ans et après 18 ans et dans chacun de ces cas, l'analyse de la régression multiple lui permet d'expliquer autour de $20 \%$ de la variance totale de ces conduites. Les prédicteurs sont différents selon le critère. Pour les conduites de violence physique entre 12 et 14 ans, ce sont, dans l'ordre, les marqueurs suivants qui sont importants: une conduite turbulente entre 8-10 ans, des résultats scolaires faibles à 11 ans, fréquenter une école où le taux de délinquance est élevé à 11 ans, être nerveux et retiré à 8-10 ans, avoir un quotient intellectuel non verbal faible à $8-10$ ans et avoir des problèmes de discipline à $8-10$ ans. En ce qui concerne les conduites violentes entre 16 et 18 ans, les prédicteurs sont, dans l'ordre de la contribution à la corrélation multiple, une délinquance cachée élevée à 14 ans, avoir eu ses premières relations sexuelles avant 14 ans, avoir quitté l'école à 15 ans, un score élevé d'agressivité à 12-14 ans, vivre dans un logement détérioré à 8-10 ans et être plus grand que la moyenne à $8-10$ ans. Finalement, la violence physique à l'âge adulte peut être prédite à l'aide des marqueurs suivants: dans l'ordre, une attitude antisociale à 18 ans, un père qui ne s'implique pas dans les activités de l'adolescent, ne pas avoir d'épargnes à 18 ans, un haut niveau de délinquance cachée à 18 ans, des revenus familiaux fáibles au milieu de l'adolescence, participer à de la violence en groupe à 18 ans et un comportement de défi à $8-10$ ans.

La prédiction de la violence physique autorapportée peut donc être entreprise avec un succès certain si les deux conditions mentionnées précédemment sont respectées: utiliser le comportement et employer des prédicteurs des quelques années précédant immédiatement l'apparition des gestes violents, surtout si l'on recourt à des marqueurs fort différents d'un âge à l'autre. Ces marqueurs, tout au moins dans le cadre de l'étude de Farrington, se classent en trois catégories : caractéristiques portant sur le milieu du sujet, intégration 
sociale et traits personnels. Voyons maintenant les résultats des études qui portent sur la violence physique enregistrée par le système de justice.

Loeber et Southamer-Loeber (1987) rapportent huit études qui présentent des résultats significatifs concernant le lien entre mentir, tricher et être agressif au moment de l'enfance et les délits ultérieurs avec violence de ces individus. D'autres travaux établissent que le faible statut socio-économique (Wikström, 1987; Hogh et Wolf, 1983) et le faible niveau d'intelligence (Hogh et Wolf, 1983 ) sont de très bon prédicteurs de la criminalité avec violence d'un individu. McCord (1988), pour sa part, établit que les variables familiales suivantes sont utiles pour prédire les délits avec violence: les conflits entre parents, une supervision inappropriée, la séparation des parents et les gestes agressifs des parents lorsque l'enfant avait entre 10 et 15 ans. Par contre les études qui se servent d'un large éventail de marqueurs sont rares. Seuls les travaux de Farrington (1989a) et les nôtres (Le Blanc et al., 1988) utilisent des variables comportementales, des milieux de vie et personnelles nombreuses.

Farrington tente de prédire les condamnations pour un délit avec violence entre le début de l'adolescence et 32 ans. Six prédicteurs s'imposent dans l'ordre suivant: peu d'intérêt pour la scolarisation à 8 ans, être défiant à 8-10 ans, avoir des parents autoritaires à 10 ans, avoir un parent qui a été condamné avant d'avoir 10 ans, être d'un poids léger à 8-10 ans et présenter un quotient intellectuel verbal faible à 8-10 ans.

Pour ce qui est de la prédiction de la délinquance officielle de nature violente après 18 ans chez les pupilles du tribunal, nous obtenons des résultats intéressants en utilisant l'analyse de la fonction discriminante (Le Blanc et al., 1988) Seules les variables de la seconde moitié de l'adolescence sont importantes; elles permettent de classifier correctement $72 \%$ des sujets (76\% de ceux qui ne commettent pas de délits avec violence et $65 \%$ de ceux qui en commettent), ce qui constitue une amélioration de $34 \%$ sur la classification au hasard de ces sujets. Dix variables permettent de réussir cette classification: dans l'ordre, la variété des délits officiels, les bagarres autorapportées, les méthodes disciplinaires punitives de la part de la mère, l'inactivité (ne pas travailler et ne pas fréquenter l'école), l'absentéisme au travail, la quantité des relations sexuelles avec des adultes, un retraitisme faible au Jesness, l'âge plus élevé des partenaires sexuels et un degré élevé d'agression autorapportée.

Compte tenu de la possibilité de prédire la présence de condamnations pour au moins un délit avec violence après 18 ans, nous nous sommes aussi demandé si nous pouvions pronostiquer le degré de violence. L'analyse de la fonction discriminante montre que les variables de la deuxième moitié de l'adolescence y arrivent encore mieux que précédemment. Le pourcentage 
de bonnes classifications est de $79 \%$ (80\% pour ceux affichant un degré faible de violence physique et $79 \%$ de ceux démontrant un degré élevé de violence), une amélioration sur la classification au hasard de ces sujets de $47 \%$. Sept variables permettent donc de séparer les criminels dont la violence est occasionnelle de ceux qui la pratiquent de façon répétitive, les chroniques. Ce sont, dans l'ordre, la variété des délits officiels, le faible retraitisme au Jesness, les méthodes disciplinaires punitives de la part de la mère, la fréquentation des débits de boisson, la fréquence des bagarres, le nombre de délits officiels avec violence à la fin de l'adolescence et la gravité totale des délits commis jusque-là.

Les variables servant à prédire la présence de délits avec violence physique sont sensiblement différentes de celles qui pronostiquent le degré de violence physique. La variété des délits connus au cours de l'adolescence domine toujours; des variables personnelles (retraitisme) et familiales (méthodes disciplinaires punitives) sont présentes à des positions dominantes; les variables comportementales sont présentes mais elles sont de nature différente, pour la présence de violence il s'agit des activités sexuelles tandis que pour le degré de violence il s'agit des conduites de violence physique; par contre, les éléments situationnels sont fort différents selon le critère; il s'agit de l'inactivité pour la présence de violence et de la fréquentation des débits de boisson pour le degré de violence. Que ce soit pour la présence ou pour le degré de violence physique officiel, il y a donc des prédicteurs qui marquent la continuité comportementale et des variables qui indiquent un degré de dysfonctionnalité de la famille et une inadaptation personnelle.

En résumé, il est possible de prédire l'apparition des gestes de violence d'une phase de la vie à l'autre, mais cette entreprise est beaucoup plus hasardeuse sur une période qui dépasse quelques années. Ainsi, de l'adolescence à la jeunesse, il est possible de prédire les actes de violence avec relativement de succès et ce qui est le plus remarquable, c'est que le prédicteur le plus puissant est la variété de l'activité criminelle au cours de l'adolescence; cette variable est ensuite supportée par des caractéristiques familiales. personnelles et situationnelles spécifiques. Par contre, il faut retenir que la prédiction de la violence physique à un âge spécifique implique ces quatres types de marqueurs, mais leur nature spécifique change substantiellement d'une phase de la vie à l'autre.

\section{QUELQUES PISTES POUR LA PRÉVENTION}

La description de la trajectoire sociale des délits avec violence au Québec nous a permis de conclure que notre société vivait depuis quelques années 
une croissance de ces délits et des batailles en groupe, mais le niveau atteint correspond à peu près à ce que nous avions connu antérieurement. Il nous est ensuite apparu que plusieurs facteurs pouvaient être responsables de cette situation: l'immigration récente, la réapparition des bandes d'adolescents et certaines transformations sociales. Ces facteurs ne seraient pas nécessairement responsables du niveau de la violence physique dans notre société, ils rendraient surtout compte de sa nature, le fait que les voies de fait dominent actuellement, et de loin, les vols qualifiés, alors que l'inverse était vrai à d'autres époques.

Par la suite, l'analyse du cheminement de l'individu qui en vient à commettre des infractions avec violence a montré qu'il s'agissait d'une activité de la jeunesse, dont la répétitivité et la durée étaient faibles et qui avait habituellement comme précurseur d'autres activités délictueuses. Il a été établi que la continuité à travers lè temps de ce type de conduite est grande, entre les gestes d'agressivité au début de l'enfance, les batailles au moment de l'adolescence et les délits avec violence à l'âge adulte. Il a finalement été établi qu'il est possible de prédire l'apparition de ce type de conduite dans la mesure où sont respectées trois conditions : utiliser prioritairement des marqueurs comportementaux, ne pas remonter trop loin dans le passé de l'individu et compléter les prédicteurs comportementaux avec des variables précises relatives à l'intégration sociale, aux caractéristiques de la personne et à des situations spécifiques qui sont génératrices de violence physique.

Ces deux niveaux d'analyse de la violence physique sont complémentaires et ils appellent des stratégies de prévention interdépendantes. Complémentaires parce que l'immigration, les bandes et les transformations sociales sont autant de contextes susceptibles de canaliser vers des modalités spécifiques de l'agir violent les individus qui sont plus enclins que d'autres à adopter des conduites agressives. Cette complémentarité comporte aussi un plan global de prévention de la violence physique. Global parce que concerné par ces deux niveaux d'action mais non pas général. Nous ne traiterons pas ici de la prévention générale de la délinquance par des interventions défensives et de développement social comme nous l'avons fait ailleurs (Fréchette et Le Blanc, 1987); nous serons plutôt concernés par la prévention spécifique des actions visant une cible limitée, la violence physique chez les adolescents et les jeunes adultes. Une prévention spécifique comportant des actions qui concernent directement des groupes et des individus susceptibles de produire des gestes impliquant de la violence physique. 


\subsection{ACTJONS SUR LES GROUPES}

Comment tenter d'altérer la trajectoire à la hausse de la violence physique? D’abord, il faut reconnaître précisément la nature de la violence physique qu'il convient de prévenir. Compte tenu que ce sont les voies de fait et les batailles entre groupes d'adolescents (plutôt que les vols qualifiés) qu'il faut avant tout réduire, les interventions défensives seront fort probablement peu utiles. Il ne s'agit pas d'augmenter la surveillance et de faire des aménagements physiques pour réduire les infractions comme dans le cas des vols à main armée dans les banques au cours des années 1970 (Gabor, Baril, Cusson, Élie, Le Blanc et Normandeau, 1987). Une surveillance policière accrue dans les transports publics et des aménagements physiques (meilleure illumination des couloirs, caméras) feront facilement diminuer les attaques dans ces lieux, mais il est aussi fort probable qu'elles se déplaceront vers d'autres lieux comme la diminution des vols de banques à Montréal s'est accompagnée d'un accroissement phénoménal des vols de dépanneurs.

Quatres voies d'actions préventives nous sont particulièrement indiquées par les facteurs que nous avons mis à jour pour orienter une stratégie globale d'intervention contre les voies de fait et les batailles entre groupes d'adolescents. Ce sont l'intégration des jeunes immigrants, le développement des mécanismes de convivialité adolescente, le soutien à la transition école-travail et une intervention énergique sur les bandes d'adolescents. D'autres interventions sont envisageables, sur l'école, la famille, les désavantages socioéconomiques et l'habitation, mais elles relèvent du développement social général, donc de la prévention générale de la délinquance et non de la prévention du phénomène spécifique de la violence physique actuelle. Même pour les voies d'interventions dont nous discuterons, nous n'avons pas la prétention d'être exhaustif en termes d'interventions possibles.

Les adolescents de parents immigrants. de première ou de deuxième génération, pour des raisons légitimes (se retrouver entre personnes qui partagent une même langue, une même culture) ou par des mécanismes d'exclusion (racisme, ghetto), tendent à se rassembier en groupes cuitureliement homogènes. Ils deviennent ainsi hautement visibles et menaçants pour la majorité et ils sont alors naturellement exclus des activités organisées pour les jeunes (activités sportives, culturelles, de convivialité). Il s'ensuit que des programmes d'intégration de ces adolescents aux activités de la majorité sont essentiels. Il faut que les services sportifs, de loisirs. les associations diverses, les maisons de jeunes fassent un effort supplémentaire pour se rendre accessibles et qu'ils aillent recruter ces adolescents de familles immigrantes.

Précédemment, nous avons mentionné que les adolescents d'aujourd hui étaient moins individualistes et qu'ils recherchaient plus la présence de leurs 
pairs, ceci, entre autres, parce que la famille devient de plus en plus restreinte et contrôlante. Une autre voie pour la prévention de la violence physique s'impose à partir de cette observation : augmenter les occasions pour que s'exerce la convivialité adolescente. Maisons de jeunes, mouvements de jeunesse, groupes sociaux, etc. sont autant de moyens de favoriser les interactions entre adolescents et ce, surtout dans un contexte ou les adultes ne sont pas absents et où l'on met l'accent sur le développement de la personne. La meilleure compréhension qui résultera de l'intégration des adolescents de familles d'immigrants à la vie sociale de l'ensemble des adolescents et qui sera aussi accentuée par une convivialité renouvelée sera le gage de la prévention des conflits interpersonnels.

La transition école-travail est plus difficile pour au moins le tiers des adolescents qui ne terminent pas leur secondaire. C'est aussi une source importance de délinquance (voir Le Blanc, 1985). Si par un effort conjoint des milieux scolaires (programmes travail-étude, programmes adaptés, etc.) et des entreprises (réserver un certain nombre d'emplois pour des jeunes), on pouvait intégrer plus de jeunes au marché du travail, il y en aurait moins d'inoccupés. Leur insertion sociale serait, comme il est bien établi, un frein puissant à l'activité délictueuse.

Une autre voie est l'intervention sur les bandes d'adolescents, surtout les bandes conflictuelles. Traditionnellement en criminologie, l'intervention consistait à assigner un travailleur de rue à un milieu donné et celuj-ci entreprenait une action sur plusieurs fronts : identifier et résoudre les conflits entre bandes, essayer de réorienter pro-socialement la bande par une action sur le leader, offrir des activités de groupe et du support individuel dans une perspective d'intégration sociale. Cette stratégie d'intervention s'est avérée totalement inefficace parce que les conflits n'ont pas nécessairement diminué, la cohésion du groupe a augmenté et les activités délictueuses aussi (voir Klein, 1971). Klein et al. (1984) proposent une stratégie à l'opposé : rendre les bandes moins attrayantes pour les membres périphériques en les surveillant et en les harcelant, neutraliser le leader (le changer d'école, l'arrêter fréquemment), rendre les batailles plus difficiles (par la surveillance, en utilisant des informateurs); il s’agit en fait de rendre leur vie de bande plus difficile.

Les quatre voies de prévention spécifique proposées - intégrer les adolescents immigrants, faciliter la transition école-travail, favoriser et orienter la convivialité adolescente et neutraliser les bandes - peuvent offrir toute une panoplie d'interventions préventives qui affecteront sûrement le niveau de violence dans notre société. Toutefois, il est un facteur qu'il ne faut pas négliger, les médias. En rapport avec ces phénomènes sociaux, ils ont souvent, de façon involontaire, un rôle d’amplificateur. Il est nécessaire de parler de 
ces phénomènes sociaux du point de vue de l'information de la population et du point de vue politique, pour que le gouvernement affecte les ressources nécessaires à la prévention de la violence physique. Mais la frontière entre en traiter trop abondamment et ainsi faire peur à la population, ou glorifier le phénomène et en parler de façon insuffisante, est souvent intangible.

\subsection{ACTIONS SUR LES INDIVIDUS}

Dans cette section concernant l'action sur les individus, nous nous limiterons, comme dans la précédente, à la prévention secondaire et spécifique. Nous traiterons de la prévention de la violence physique chez les individus à risque et nous laisserons de côté le traitement des individus qui ont déjà passé à l'acte ou qui ont été reconnus comme des personnes qui commettent des délits avec violence. Commençons par la question du dépistage et ensuite voyons quelles seraient les pistes pour l'intervention préventive, ceci pour divers critères à différents âges.

Le dépistage des individus à risque est possible dans la mesure où les comportements cibles sont définis avec précision et si l'on considère les marqueurs appropriés. Considérons d'abord le cas des enfants de 8-10 ans qui se bagarreront régulièrement entre 12 et 14 ans; ils peuvent être identifiés grâce aux informations suivantes: présenter un comportement turbulent et des problèmes de discipline, avoir peu de succès à l'école et fréquenter une école dont le taux de délinquance est élevé. Au moins trois types d'interventions préventives sont en conséquence indiqués: des programmes de soutien pour contrer la démotivation et la marginalisation scolaire de ces sujets qui se consolident au début du secondaire et des interventions pour améliorer la qualité de l'école et réduire son niveau de délinquance (voir Côté, Le Blanc, Ouellet et Pronovost, 1988, pour un inventaire des programmes possibles) et aussi des actions auprès de la famille pour qu'elle reprenne en main la discipline (voir Patterson, 1982).

Si l'on pense maintenant à ceux qui se bagarreront régulièrement entre 16 et 18 ans, ils peuvent être dépistés à 14 ans d'une façon relativement efficace. Les informations utiles pour le faire sont les suivantes: manifester un degré élevé de délinquance cachée, avoir quitté ou être sur le point de quitter l'école, présenter un degré élevé d'agressivité, avoir des amis délinquants et provenir d'un milieu défavorisé. Cette liste de marqueurs pointe vers deux types d'interventions préventives: la transition école-travail et la neutralisation de l'influence des pairs délinquants. Aussi les programmes qui faciliteront l'intégration au marché du travail de ces adolescents mal préparés d'un milieu défavorisé sont essentiels ainsi que les interventions qui réduiront l'influence 
des pairs délinquants par l'intégration à des groupes d'adolescents pro-sociaux (voir par exemple Feldman, Caplinger et Wodarski, 1983).

En dernier lieu, si nous adoptons comme cible ceux qui commettront des délits avec violence après 18 ans, les prédicteurs se retrouvent à la fin de l'adolescence. Il s'agit d'abord d'un degré élevé de délinquance officielle et de la tendance à se battre, d'être originaire d'un milieu défavorisé et d'être inactif sur le marché du travail, de provenir d'une famille où les méthodes disciplinaires étaient punitives et dont le père n'était pas engagé dans la vie familiale, d'adopter un style de vie qui suppose des risques d'altercations (fréquenter régulièrement des débits de boisson, avoir des activités sexuelles avec des partenaires diverses et plus vieilles) et d'afficher des attitudes antisociales. Certains de ces marqueurs peuvent constituer des indices utiles au dépistage mais ils ne peuvent offrir des points d'ancrage pour l'intervention, par exemple, les caractéristiques passées de la famille alors que le jeune adulte s'en détache. Les autres prédicteurs indiquent des voies d'interventions préventives que nous avons déjà mentionnées, intégration au marché du travail et limitation de l'influence des pairs asociaux. Un dernier type d'action apparaît toutefois pertinent pour ceux qui font partie de ce groupe: les aider à trouver des activités et des groupes pro-sociaux pour remplacer les pairs asociaux et la fréquentation des débits de boisson.

Qu'il s'agisse des jeunes de la première ou de la seconde partie de l'adolescence ou des adultes au moment de la jeunesse, les marqueurs renvoient à des interventions préventives relatives à l'intégration sociale et à la neutralisation de l'influence des pairs asociaux, mais puisque les comportements d'agression semblent persister dans le répertoire comportermental de l'individu sous diverses formes selon l'âge, peut-être que des actions de nature psychologique seraient essentielles. Des actions de rééducation sur les façons de penser et de réagir aux stimulations déplaisantes: ces types d'interventions plus fondamentales ne seraient pas seulement utiles mais aussi nécessaires comme le montrent Mathias, DeMuro et Allinson (1984).

En terminant cette section, il convient de noter la convergence que nous imposent les facteurs de la montée de la violence physique et les marqueurs de l'utilisation probable de la violence par les individus. D'un côté comme de l'autre, la priorité revient naturellement aux programmes qui favorisent l'intégration sociale, la réinsertion dans des groupes pro-sociaux d'activités de travail, de récréation et de convivialité, et qui garantissent la neutralisation des groupes et des individus asociaux. Et les mécanismes pour atteindre ces objectifs sont le développement social intégré et le développement personnel différentiel. 


\section{RÉFÉRENCES}

BRAKE, M. (1985), Comparative Youth culture: The sociology of Youth Cultures and Youth Subcultures in America, Britain and Canada, London. Routledge \& Kegan Paul.

CAMPBELL, A. (1984), The girls in the gangs, New York, Basil Blackwell.

CHARLEBOIS, P., LE BLANC, M., TREMBLAY, R.E., GAGNON, C., LARIVÉE, S. (en préparation), Early Innocuous Disruptive Behaviors and Later Self-reported Delinquency.

CLOWARD, R.A., OHLIN, L.E. (1960), Detinquency and opportunity: a theory of delinquent gangs, Gleancoe, The Free Press.

COHEN, A.K. (1955), Delinquent Boys: the Culture of the Gang, Glencoe, The Free Press.

COHEN, S. (1973), Folk Devils and Moral Panics: The Creation of the Mods and Rockers. St-Albans, Paladin.

CONSEIL DES AFFAIRES SOCIALES (1989), Deux Québec dans un : rapport sur le développement social et démographique, Montréal, Gaétan Morin. Gouvernement du Québec.

CÔTÉ, G., LE BLANC, M., OUELLET, G., PRONOVOST, A. (1988), Violence er délinquance à l'école : inventaire de mesures préventives, Montréal, Commission des écoles catholiques de Montréal. Service des études.

CUSSON, M. (1990), Croissance et décroissance du crime, Paris, Presses universitaires de France.

Direction générale de la sécurité publique, Direction de la recherche et du développement, ministère de la justice, Statistiques 1986-1987-1988.

FARRINGTON, D.P. (1989a), «Early Predictors of Adolescent Aggression and Adult Violence", Violence and Victims, 4.

FARRINGTON, D.P. (1989b), «Childhood Aggression and Adult Violence: Early Predictors and Later Life Outcomes", in K.H. RUBIN AND D. PEPLER. The devetopment and treatment of childhood aggression, Hilsdale, Lawrence Erlbaum.

FELDMAN, R.A., CAPLINGER, T.E., WODARSKY, J.J. (1983). The Saint Louis conundrum: the effective treatment of antisocial youth, Englewood Cliffs, Prentice hall.

FRÉCHETTE, M., LE BLANC, M. (1987), Délinquances et délinquants, Chicoutimi. Gaétan Morin.

GABOR, T, , BARIL, M., CUSSON, M. ÉLIE. D., LE BLANC, M., NORMANDEAU. A. (1987), Armed Robbery: Cops, Robbers and Victions. Soringfield, Charles C. Thomas.

HAMPARIAM, D.M., DAVIS, J.M., JACOBSON, J.M., McGRAW, R.E. (1985), The young criminal years of the violent few, Washington, National Institute for Juvenile Justice and Delinquency Prevention.

HÉBERT, J. (1989), «La problématique des jeunes agressifs: des points de repère". Apprentissage et socialisation en piste. 12, 1: 45-52.

HOGH, E., WOLF, P. (1983), "Violent crime in a Birth Cohort: Copenhagen 1953-1977», in K.T. VAN DEUSEN ET S.A. MEDNICK, Prospective studies in crime and delinquency. Boston, Kluwer-Nijhoff. 
HUESMAN, L.R., ERON, L.D., LEFKOWITZ, M.M., WALDER, L.O. (1984), «Stability of Aggression Over Time and Generations», Developmental psychology, 20, 6: $1120-1134$.

KLEIN, M.W. (1971), Street Gangs and Street Workers, Englewood Cliffs, Prentice Hall.

KLEIN, M.W., MAXSON, C.L., GORDON, M.A. (1984), Evaluation of an Imported Gang Violence Deterrence Program. Social science institute, University of Southern California.

LANGELIER-BIRON, L. (1989), "La violence dans le Québec des années 1980», Apprentissage et socialisation en piste, 12, 1 : 37-44.

LE BLANC, M. (1971), Drogue-jeunesse: Montréal, été 1970, Ottawa, Commission d'enquête sur l'usage des drogues à des fins non médicales.

LE BLANC, M. (1977), La délinquance juvénile au Québec, Québec, Éditeur officiel du Québec, ministère des Affaires sociales.

LE BLANC, M. (1985), «L'école, un mécanisme amplificateur de la délinquance des adolescents", in M. CRESPO ET C. LESSARD, Éducation en milieu urbain, Montréal, Presses de l'Université de Montréal.

LE BLANC, M., CÔTÉ, G. (1986), «Comparaison des adolescents de 14-15 ans en 1974 et en 1985", in R.E. TREMBLAY, M. LE BLANC, A.E. SCHWARTZMAN, $L a$ conduite délinquante des adolescents de Montréal (1974-1985): étude descriptive et prédictive, Montréal, École de psycho-éducation, Université de Montréal.

LE BLANC, M., HÉBERT, C., DAVID, P. (1988), Prédiction de l'inadaptation à l'âge adulte, Centre international de criminologie comparée, Université de Montréal.

LE BLANC, M., FRÉCHETTE, M. (1989), Male Criminal Activity From Childhood Through Youth: Multilevel and Developmental Perspectives, New York, Springer-Verlag.

LOEBER, R. (1985), «Patterns and Development of Antisocial Child Behavior», in G.J. WHITEHURST, Annals of Child development, 2: 138-166, Greenwich, JAl Press.

LOEBER, R., SOUTHAMER-LOEBER, M. (1987), «Prediction», in H.C. QUAY, Handbook of juvenile delinquency, New York, John Wiley \& Sons.

LOEBER, R., LE BLANC, M. (1990), «Toward a Developmental Criminology», in M. TONRY, N. MORRIS, Crime and justice: an annual review, 13: 1-98. Chicago, University of Chicago Press.

MATHIAS, R.A., DEMURO, P., ALLINSON, R.S. (1984), Violent Juvenile Offender: An Anthology, San Francisco, National Council on Crime and Delinquency.

McCORD, J. ( 1988), «Parental Behavior in the Cycle of Aggression», Psychiatry, 51 : 14-23.

MILLER, W.B. (1975), Violence By Youth Gangs and Youth Groups as a Crime Problem in Major American Cities, Washington, National Institute for Juvenile Justice and Delinquency Prevention.

MILLER, S.J., DINITZ, S., CONRAD, J.P. (1982), Careers of the Violent : The Dangerous Offender and Criminal Justice. Lexington, Lexington Books.

OLWEUS, D. (1979), «Stability of Aggressive Reaction Patterns in Male: A Review». Psychological Bulletin, 86: 852-875.

PATTERSON, G.R. (1982), A Social Learning Approach, vol, I: Carcive Family Process. Eugene, Castalia. 
RACINE, A., SOMERHAUSEN, C., DEBUYST, C., DE BOCK, G., DE BRAY, L. (1966), Les blousons noirs: un phénomène socio-culturel de notre temps, Paris, Cujas.

REISS, A. (1988), "Co-offending and Criminal Careers", in M. TONRY AND N. MORRIS, Crime and Justice: An Annual Review, 10:117-170, Chicago, University of Chicago Press.

RICE, B. (1977), "The New Gangs of Chinatown», Psychology Today, May: 60-69.

ROBERT, P., LASCOUMES, P. (1974), Les bandes d'adolescents : une théorie de la ségrégation, Paris, Les éditions ouvrières.

ROFF, M., WIRT, R.D. (1984), "Childhood Aggression and Social Adjustment as Antecedents of Delinquency», Journal of Abnormal child psychology 12: 11-26.

ROY, J. (1989), «L'inévitable violence», Apprentissage et socialisation en piste, 12, 1 : 29-36.

SHORT, J.F., STRODTBECK, F.L. (1965), Group Process and Gang Delinquency, Chicago, University of Chicago Press.

THRASHER, F.M. (1927), The gang: A Study of 1318 Gangs in Chicago, Chicago, University of Chicago Press.

TREMBLAY, R.E., DESMARAIS-GERVAIS, L. (1986), «La prédiction de l'inadaptation de l'enfance à l'adolescence», in R.E. TREMBLAY, M. LE BLANC, A.E. SCHWATZMAN, La conduite délinquante des adolescents à Montréal (1974-1985); étude descriptive et prédictive, Montréal, Université de Montréal.

TREMBLAY, R.E., LE BLANC, M., SCHWARTZMAN, A. (1988), "The Predictive Power of First Grade an Teacher Rating: Sex Differences in Antisocial Behavior and Personnality at Adolescence». Journal of Abnormal Child psychology, 16, 3:571-583.

VIGIL, J.D. (1988), Barrio Gangs: Street Life and Identity in Southern California, Austin, Texas University Press.

WHITE, W.F. (1943), Street Corner Society: The Social Structure of an Italian Slum. Chicago, University of Chicago Press.

WIKSTRÖM, P-O.H. (1985), Everyday Violence in Contemporary Sweden: Situational and Ecological Approach, Stockholm, The National Council for Crime Prevention, Report, $\mathrm{n}^{\circ} 15$.

WIKSTRÖM, P-O.H. (1987), Patterns of Crime in a Birth Cohort, Stockholm, University of Stockholm, Department of Sociology.

WOLFGANG, M.E., THORNBERRY, T.P., FIGLIO, R.M. (1987), From Boy to Man, from Delinquency to Crime, Chicago, University of Chicago Press.

YABLONSKY, L. (1962), The Violent Gang, Baltimore, Penguin Books. 